\title{
Optimizing Two-Stage Modular Wastewater Plant Expansions Using Numerical Methods and Simulation in A Real Options Context
}

\author{
Y. Lawryshyn ${ }^{1}{ }^{*}$, B. Zhang ${ }^{1}$, and M. Davison ${ }^{2}$ \\ ${ }^{1}$ Department of Chemical Engineering and Applied Chemistry, University of Toronto, Toronto, M5S 3E5, Ontario, Canada \\ ${ }^{2}$ Departments of Applied Mathematics, Western University Canada, London, N6A 5B7, Ontario, Canada
}

Received 10 October 2020; revised 17 November 2020; accepted 19 November 2020; published online 31 December 2020

\begin{abstract}
The optimization of a modular expansion strategy, while extremely relevant in the industrial setting, requires sophisticated numerical modeling for the valuation of even simple scenarios. In this work, we develop both a numerical model and a model based on Monte-Carlo simulation utilizing real options, to provide a methodology for optimizing a plant expansion strategy. Our case study is associated with a wastewater treatment plant expansion; however, the methodologies developed here can be extended to many industrial settings, including mining, oil and gas, and manufacturing. The value of the Monte-Carlo simulation is that it is much more easily understood by practitioners and more versatile in that it can be used to model non-standard processes. The results of both of our models match consistently, essentially validating the Monte-Carlo technique.
\end{abstract}

Keywords: real options, optimization, modular expansion, wastewater treatment, Monte-Carlo simulation

\section{Introduction}

Decision makers are often faced with the task of deciding on the design capacity of capital asset investment projects. Typically, discounted cash-flow (DCF) models are used for analysis leading to rough estimates of optimal asset size. The ability for managers to react to uncertainties at a future time adds value to projects, and since this value is not captured by standard DCF methods, erroneous decision making may result (Trigeorgis, 1996). Real option analysis (ROA) is recognized as a superior method to quantify the value of real-world investment opportunities where managerial flexibility can influence their worth, as compared to standard DCF methods. ROA stems from the work of (Black and Scholes, 1973) on financial option valuation. Myers (1977) recognized that both financial options and pro-ject decisions were exercised after uncertainties were resolved. Early techniques therefore applied the Black-Scholes equation directly to value put and call options on tangible assets (Brennan and Schwartz, 1985). Since then, ROA has gained significant attention in academic and business publications, as well as textbooks (Trigeorgis, 1996; Copeland and Antikarov, 2001). An excellent empirical review of expost investment decisions made in copper mining showed that fewer than half of investment timing decisions were made at the right time and 36 of the 51 pro-

${ }^{*}$ Corresponding author. Tel.: +1-416-946-0576; fax: +1-416-978-8605.

E-mail address: yuri.lawryshyn@utoronot.ca (Y. Lawryshyn).

ISSN: 2663-6859 print/2663-6867 online

(c) 2020 ISEIS All rights reserved. doi:10.3808/jeil.202000043. jects analyzed should have chosen an extraction capacity of $40 \%$ larger or smaller (Auger and Guzman, 2010). The authors were unaware of any mining firm basing all or part of their decision making on the systematic use of ROA and emphasize that the "failure to use ROA to assess investments runs against a basic assumption of neoclassical theory: under uncertainty, firms ought to maximize their expected profits". They make the case that irrational decision making exists within the industry due to a lack of real option tools available for better analysis. A recent review of the use of real option analysis for infrastructure projects concluded that the use of real options arises due to the need for a new approach to infrastructure management and valuation, since the DCF method does not allow one to capture the value of flexibility (Martins et al., 2015). In their work, they evaluate a number of leading approaches used for real options valuation. They note a number of advantages of using Monte-Carlo simulation for ROA as the approach is considered to be user-friendly and provides results that are simple to explain to the decision makers. Other studies also support the use of Monte Carlo simulation in ROA (Benedetti et al., 2005; Ferrer et al., 2008).

In this paper, we consider the problem of optimizing the size of a modular wastewater treatment plant. As one of the most capital-intensive industrial sectors in North America, the environmental water and wastewater sector faces a significant need for near future capital investment. According to the Federation of Canadian Municipalities, in 2007, a total of $\$ 31$ billion was needed for refurbishment of existing systems and $\$ 57$ billion was needed for replacing existing systems and constructing new ones. 


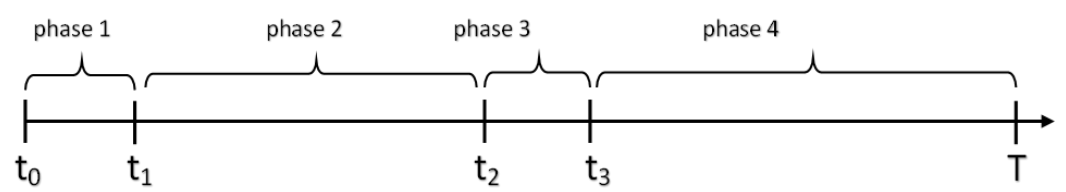

Figure 1. Depiction of the timelines for the plant expansion.

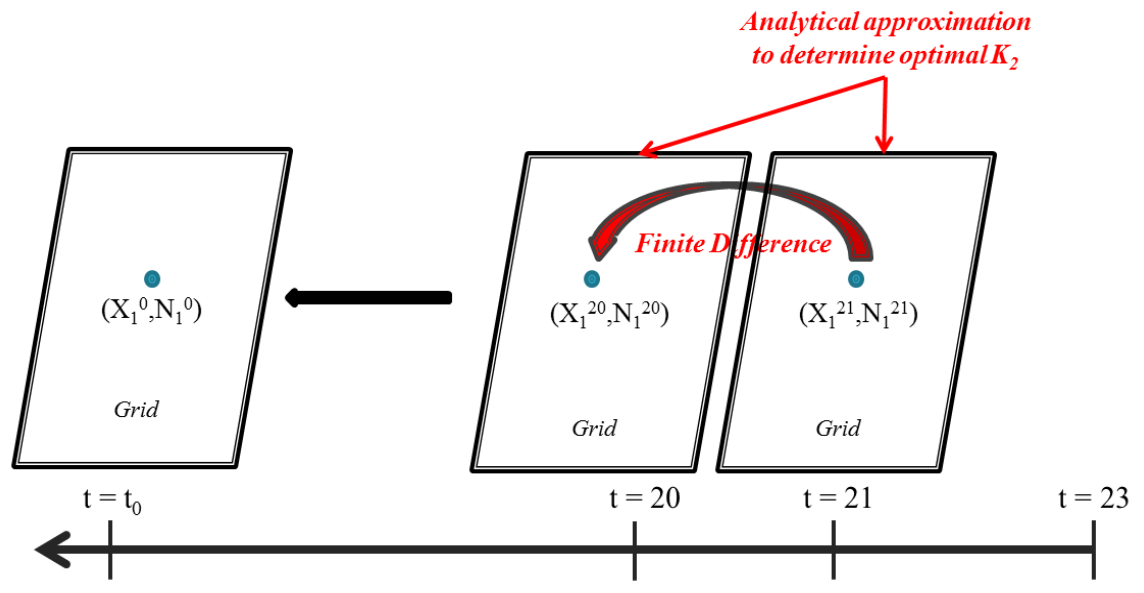

Figure 2. Depiction of the numerical model.

The Conference Board of Canada reported that the average capital expenditures in municipal water and wastewater have reached $\$ 1.5$ billion annually from $1998 \sim 2006$, with the capital expenditures in 2006 being close to $\$ 2.4$ billion for the whole country (Public-private partnerships in Canada, 2013). Although some advanced water treatment technologies have been developed and put into use in municipal systems, capital and research expenditures are being held back in the sector due to restricted margins and regulated pricing leading to suboptimal returns (Global Cleantech Center, 2013). More recently, the Canadian Infrastructure Report Card (CIRC, 2019) emphasized that the state of the Canadian infrastructure is at risk with a majority of the infrastructure that Canadians rely on every day being more than 20 years old. Clearly, better capital asset allocation strategies in the water and wastewater sector can significantly improve capital expenditure efficiency and allow more capital to be invested in the research and development of better treatment technologies.

A single stage "real option" approach that copes with future demand uncertainties for wastewater treatment plant expansion was first developed by (Lawryshyn and Jaimungal, 2010), who modeled the growth in demand for a residential wastewater treatment as a geometric Brownian motion (GBM) that is partially correlated to an appropriate traded market index. Their model transformed expected future penalty costs for wastewater connections as an asian option with the expansion size being the strike price. The finite difference method was used to numerically solve for the capital needed to fund the single stage expansion. Lawryshyn and Jaimungal further developed a closed form approximation to the modular expansion problem. The modular expansion model allows a second-time expansion of the plant at a pre-defined future date. Their results showed that a modular expansion requires significantly less up-front capital investment, and the overall expected expenditures were reduced compared to the single-stage expansion model (Lawryshyn and Jaimungal, 2014). However, the modular expansion model developed by Lawryshyn and Jaimungal can only account for a single expansion at a pre-fixed future date, which limits the applicability of the model in practice.

In the model developed here, the pre-fixed expansion date is relaxed, i.e., the second stage expansion is modeled in such a way that it can be carried out at any point after initial expansion. This is accomplished by utilizing a combination of the finite difference method and the analytical approximation developed by (Lawryshyn and Jaimungal, 2010). The result is that a more realistic future expansion strategy is modeled. However, the resulting model may still be considered somewhat mathematical in nature and too complicated for practicing engineers/decision makers. Thus, a practical, easy to understand Monte-Carlo simulation approach was also developed. An important adventage of the Monte-Carlo model is its flexibility to model other stochastic processes regarding the plant demand. Results obtained by using both methods are compared and found to be similar to each other.

\section{Materials}

In this section, we present the methodology utilized in our models. First, we present the project background, which has its roots in the work of Lawryshyn and Jaimungal (Lawryshyn and Jaimungal, 2010, 2014). Next, we formulate our model utilizing a partial differential equation (PDE) coupled with an analytical approximation to develop the valuation. In the last section we present 
our model using Monte-Carlo simulation. While the simulation relies on least squares Monte-Carlo(LSMC) techniques, the model is much more intoitive and will likely be more readily accepted within the industry.

\subsection{Project Background}

The objective of the model presented in this paper is to determine the optimal sizing of a wastewater treatment plant (WWTP) for a municipality that is undergoing high but uncertain growth. Currently, the existing plant has limited capacity and a decision is required regarding the size of the current expansion and the maximum total potential capacity after a second, future expansion, is made. Plant construction is expected to take just under 2 years and the total plant life is estimated to be 25 years. An undersized plant will lead to significant overflow penalty costs while an oversized plant may show poor performance and will also require significant up front capital costs that may be difficult to recoup if significant population expansion is not realized. Clearly, a staged design of the plant expansion can help the municipality to reduce the cost of an initial over-design yet still allow for the option to expand, if significant population growth is realized.

There are four phases involved in the two-stage expansion of a WWTP project (see Figure 1):

- Phase 1: a decision on the first module size will be made at time $t_{0}$ and construction will take place for the first modular plant from time $t_{0}$ to time $t_{1}$.

- $\quad$ Phase 2: the operation of the first modular plant starts from time $t_{1}$; after time $t_{1}$, the municipality has the option to expand the wastewater treatment plant at any time $t_{2}$ if necessary.

- $\quad$ Phase 3: if necessary, construction of the second module will begin at $t_{2}$, ending at $t_{3}$.

- $\quad$ Phase 4: the operation of the second module will take place from time $t_{3}$ to $T$.

Following (Lawryshyn and Jaimungal, 2010 and 2014), we assume that the expansion rate fol-lows a GBM, that is correlated to a traded security (market index). The price of the security also follows a GBM process:

$d S_{t}=\mu_{S} S_{t} d t+\sigma_{S} S_{t} d W_{t}$

where $\mu_{s}$ and $\sigma_{s}$ are constants representing the drift and volatileity, respectively and $W_{t}$ is a Wiener process. Similarly, the connection rate (expansion rate) is modeled as:

$$
d X_{t}=\mu_{X} X_{t} d t+\sigma_{X} X_{t}\left(\rho d W_{t}+\sqrt{1-\rho^{2}} d W_{t}^{\perp}\right)
$$

where $\mu_{X}$ is the drift and $\sigma_{X}$ is the volatility, $\rho$ is the correlateon and $W_{t}^{\perp}$ is another Wiener process, independent of $W_{t}$. Under the risk-neutral measure, the connection rate becomes:

$$
d X_{t}=\bar{r} X_{t} d t+\sigma_{X} X_{t}\left(\rho d \tilde{W}_{t}+\sqrt{1-\rho^{2}} d W_{t}^{\perp}\right)
$$

where:

$$
\bar{r}=\mu_{X}-\frac{\rho \sigma_{X}}{\sigma_{S}}\left(\mu_{S}-r\right)
$$

$r$ is the risk-free rate and $\tilde{W}_{t}$ is an equivalent process to $W_{t}$ under the risk-neutral measure. The market parameters are presented in Table 1.

The total number of connections at time $t$ is given by:

$N_{t}=N_{0}+\int_{0}^{t} X_{u} d u$

And defining the penalty cost associated with a lack of capacity as:

$P C_{t}=\max \left(0,\left(N_{t}-K\right) \cdot P C_{0}\right)$

Leads to the following present value of the penalty cost incurred from time $t$ to $T$ :

$\tilde{\mathbb{E}}\left[P C_{t, T ; K}^{P V}\right]=\int_{t}^{T} P C_{0} e^{-r u} \cdot \tilde{\mathbb{E}}\left[\left(N_{0}+\int_{0}^{u} X_{s} d s-K\right)_{+}\right] d u$

where $P C_{0}$ is the penalty cost rate associated with insufficient plant capacity per connection, and $K$ is the size of the plant expansion. (Lawryshyn and Jaimungal, 2014) provided an analytical approximation for Equation (7) as:

$\tilde{E}\left[P C_{t, T ; K}^{P V}\right] \sim$
$\int_{t}^{T} P C_{0} e^{-r u} \cdot e^{\tilde{\mu}_{0, u}}\left(X_{0} \Phi\left(d_{0, u ;+}\right)-\left(K-N_{0}\right) e^{-\tilde{\mu}_{0, u}} \Phi\left(d_{0, u ;-}\right)\right) d u$

where $\Phi$ is the standard normal cumulative distribution function:

$d_{t, T ; \pm}=\frac{\ln \left(X_{t} / K_{t}^{\prime}\right)+\tilde{\mu}_{t, T} \pm \frac{1}{2} \tilde{\sigma}_{t, T}^{2}}{\tilde{\sigma}_{t, T}}$

$K_{t}^{\prime}=K-N_{t}$

$\tilde{\mu}_{t, T} \equiv \tilde{\mu}=\ln \left(e^{\bar{r}(T-t)}-1\right)-\ln \bar{r}$

And:

$$
\tilde{\sigma}_{t, T} \equiv \tilde{\sigma}=\sqrt{\begin{array}{l}
\ln \left(\frac{2}{\bar{r}+\sigma_{X}^{2}}\right) \\
+\ln \left(\frac{e^{\left(2 \bar{r}+\sigma_{X}^{2}\right)(T-t)}-1}{2 \bar{r}+\sigma_{X}^{2}}-\frac{e^{\bar{r}(T-t)}-1}{\bar{r}}\right) \\
+2 \ln \bar{r}-2 \ln \left(e^{\bar{r}(T-t)}-1\right)
\end{array}}
$$



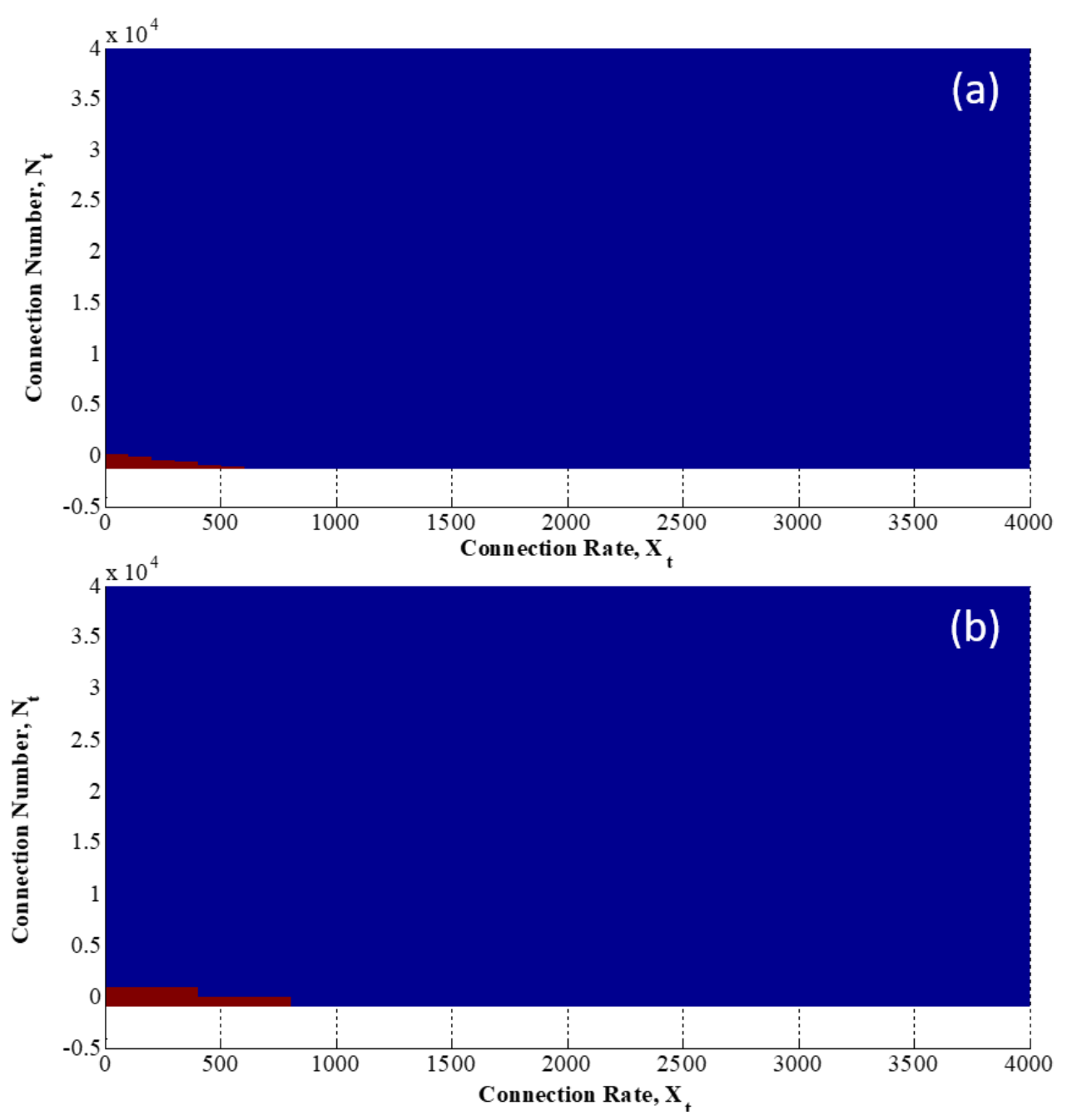

Figure 3. Construction boundary plots for the Numerical Model (a) and the Monte-Carlo Simulation (b) at $t=22 y$. Blue designates construct.

Table 1. Market Parameters

\begin{tabular}{ll}
\hline Parameter & Value \\
\hline Market drift, $\mu_{S}$ & $10 \%$ \\
Market volatility, $\sigma_{S}$ & $16 \%$ \\
Connection rate drift, $\mu_{X}$ & $8 \%$ \\
Connection rate volatility, $\sigma_{X}$ & $5 \%$ \\
Correlation factor, $\rho$ & 0.5 \\
Risk free rate, $r$ & $2 \%$ \\
\hline
\end{tabular}

Table 2. Construction Cost Parameter Values

\begin{tabular}{ll}
\hline Parameter & Value \\
\hline Module 1 fixed cost, $\alpha_{1}$ & $\$ 3,500,000$ \\
Module 2 fixed cost, $\alpha_{2}$ & $\$ 525,000$ \\
Module 1 variable cost 1, $\gamma_{f}$ & $\$ 860 /$ connection \\
Module 1 variable cost 2, $\gamma_{12}$ & $\$ 172 /$ connection \\
Module 2 variable cost, $\gamma_{2}$ & $\$ 757 /$ connection \\
\hline
\end{tabular}

Or, in the case where we are interested in a filtration $\mathcal{F}_{t}$, where $\tau \leq t$, Equation (8) can be written as:

$$
\begin{aligned}
& \tilde{E}\left[P C_{t, T ; K}^{P V} \mid \mathcal{F}_{t}\right] \sim \\
& \int_{t}^{T} P C_{0} e^{-r u} \cdot e^{\tilde{\mu}_{\tau, u}}\left(X_{\tau} \Phi\left(d_{\tau, u ;+}\right)-\left(K-N_{\tau}\right) e^{-\tilde{\mu}_{\tau, u}} \Phi\left(d_{\tau, u ;-}\right)\right) d u
\end{aligned}
$$

Lawryshyn and Jaimungal (2014) modeled the expansion costs with fixed and variable components as:

$$
C_{1}\left(K_{1}, K_{2 \max }\right)=\alpha_{1}+\gamma_{1} K_{1}+\gamma_{12} K_{2 \max }
$$

For the first expansion and:

$$
C_{2}\left(K_{2} \leq K_{2 \max }\right)=\alpha_{2}+\gamma_{2} K_{2}
$$

For the second, where $K_{l}$ is the size of the first expansion, $K_{2}$ is the size of the second expansion, $K_{2 \max }$ is the maximum size for the second expansion and $\alpha_{1}, \alpha_{2}$ are positive coeffi- 

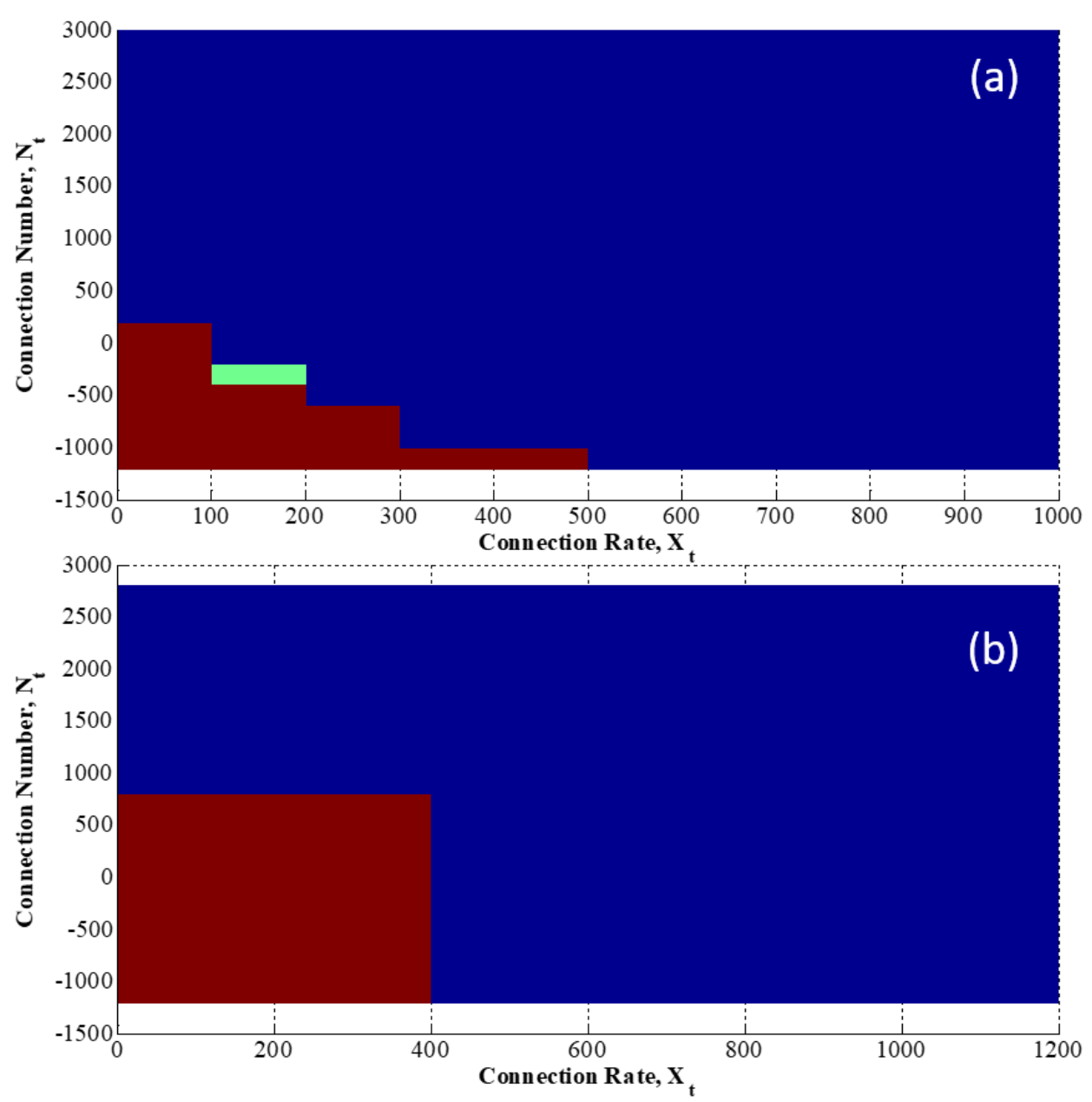

Figure 4. Construction boundary plots for the Numerical Model (a) and the Monte-Carlo Simulation (b) at $t=2 y$. Blue designates construct.

cients associated with fixed construction cost, and $\gamma_{1}, \gamma_{2}$ and $\gamma_{12}$ are positive coefficients associated with variable construction cost. The cost parameter values are presented in Table 2. Throughout the models, $X_{0}$ is assumed to be 81 connections per year and $N_{0}$ is assumed to be -200 connections (i.e., the current plant capacity is for 200 connections).

Lawryshyn and Jaimungal (2014) showed that at some time $t^{*}$ the optimal second state expansion size can be determined by:

$$
K_{2}^{O p t}=\min _{K_{2}}\left(C_{2}\left(K_{2}\right)+\tilde{E}\left[P C_{t^{*}+t_{\text {const }}, T ; K_{1}+\min \left(K_{2}, K_{2 \max }\right)}^{P V} \mathscr{F}_{t^{*}}\right] e^{-r t^{*}}\right)
$$

where $t_{\text {const }}$ is the time required for construction of the expansion. Equation (16) will be used extensively in the numerical model for determining the optimal strategy at each decision point. It should be noted that Equation (16) could also be used in the Monte-Carlo simulation to reduce computational time, however in view of the fact that one of the objectives was to develop a model purely based on Monte-Carlo simulation, a technique much more readily accepted by practitioners, a different opti- mization algorithm was utilized. In the following subsection, the numerical model methodology is presented and in the subsequent one, the Monte Carlo model is developed.

\subsection{Numerical Model}

Defining $v_{t}$ as the total cost of the plant and recognizing that $v\left(t, X_{t}, N_{t}\right)$, applying Ito's lemma and standard arguments leads to the following PDE:

$\frac{\partial v}{\partial t}+\bar{r} x \frac{\partial v}{\partial x}+x \frac{\partial v}{\partial y}+\frac{1}{2} \sigma_{x}^{2} x^{2} \frac{\partial^{2} v}{\partial x^{2}}=r v$

where the dummy variables $x$ and $y$ were used to replace $X_{t}$ and $N_{t}$, respectively. We note that the same PDE was developed in (Lawryshyn and Jaimungal, 2010), however, the boundary conditions in this case are different. Specifically, we assume constant slope as follows:

$\left.\frac{\partial v}{\partial x}\right|_{x=x_{\max }}=$ constant 


$$
\begin{aligned}
& \left.\frac{\partial v}{\partial y}\right|_{y=y_{\max }}=\text { constant } \\
& \left.\frac{\partial v}{\partial y}\right|_{y=y_{\min }}=\text { constant }
\end{aligned}
$$

And,

$$
v(t, 0, y)=P C_{0} e^{-r u} \tilde{E}\left[\max \left(N_{t}-K, 0\right)\right] \cdot(T-t)
$$

As discussed previously, we assume that the managers have the option to expand the plant at any time after initial construction is completed during the life of the plant and decisions are made on an annual basis only. However, since the construction time of the second expansion is just under two years, it makes no sense to expand at year $24^{4}$. Thus, a decision regarding the size of the modular expansion is required at times 2 years to 23 years. Thus, at $t=23$, we utilize Equation (16) to determine the optimal expansion size, $K_{2}^{O p t, t=23}$. Next, we need to compare the cost of constructing and incurring the new (reduced) penalty cost where the plant size is now $K_{1}+K_{2}^{O p t, t=23}$ versus not constructing and incurring the penalty cost given the plant size of $K_{l}$. Utilizing Equations (13) and (15) we can calculate the expansion strategy cost at $t=23$ as:

$$
C_{t=23}^{E S}\left(X_{t}, N_{t}\right)=C_{2}\left(K_{2}^{O p t, t=23}\right)+\tilde{\mathbb{E}}\left[P C_{t=23, T ; K_{1}+K_{2}^{O p t, t=23}}^{P V}\right]
$$

The no construction cost at $t=23$ is simply:

$$
C_{t=23}^{N C}\left(X_{t}, N_{t}\right)=\tilde{\mathbb{E}}\left[P C_{t=23, T ; K_{1}}^{P V}\right]
$$

And therefore for the boundary condition for $v$ becomes:

$$
v\left(t=23, X_{t}, N_{t}\right)=\min \left(C_{t=23}^{N C}\left(X_{t}, N_{t}\right), C_{t=23}^{E S}\left(X_{t}, N_{t}\right)\right)
$$

The next decision time point is at year 22, thus we utilize Equation (17) to determine $v\left(t=22, X_{t}, N_{t}\right)$. At this point, we compare the expansion strategy cost to $v$ as of this time. Thus, we have:

$$
C_{t=22}^{E S}\left(X_{t}, N_{t}\right)=C_{2}\left(K_{2}^{O p t, t=22}\right)+\tilde{\mathbb{E}}\left[P C_{t=22, T ; K_{1}+K_{2}^{O p t, t=22}}^{P V}\right]
$$

And:

$C_{t=22}^{N C}\left(X_{t}, N_{t}\right)=v\left(t=22, X_{t}, N_{t}\right)$

And $v$ is updated as:

$$
v\left(t=22, X_{t}, N_{t}\right)=\min \left(C_{t=22}^{N C}\left(X_{t}, N_{t}\right), C_{t=22}^{E S}\left(X_{t}, N_{t}\right)\right)
$$

The methodology is repeated until $t=2 y$, after which
Equation (17) is utilized to find the cost at $t=0$. This procedure was run with different $K_{I}$ and $K_{2 \max }$ values to determine the optimal initial construction size and maximum modular expansion size. A depiction of the numerical model is presented in Figure 2.

\subsection{Monte-Carlo Simulation}

The Monte Carlo simulation methodology requires that we form a grid of starting values for $X_{t}$ and $N_{t}$ at each decision making time point, i.e., as before, $t=2,3 \ldots, 23$ years. Starting at $t$ $=23 y$, for each grid point $(i, j)$ we simulate $X_{t}$ from $t=23 y$ to $t=T$, for $N$ paths and thus we arrive at a $M_{\text {path }} \times M_{\text {time }}$ matrix, where $M_{\text {path }}$ is the number of paths (per grid point) and $M_{\text {time }}$ is the number of time steps for the simulation (at $t=23$ we would be simulating until $t=T=25 y$, so $\left.M_{\text {time }}=(25-23) / d t\right), X_{t=23, T}^{i, j}$. Integrating, it is then possible to determine the number of new connections, $\boldsymbol{N}_{t=23, T}^{i, j}$, where the $k$-th, $l$-th element of this matrix is given by:

$$
\left.\boldsymbol{N}_{t=23, T}^{i, j}\right|_{k, l}=N_{t=23}^{i, j}+\left.\sum_{m=1}^{l} \boldsymbol{X}_{t=23, T}^{i, j}\right|_{k, m} d t
$$

And where $d t$ is the time step in the simulation. Thus, for any total plant size $K$ the present value of the penalty cost for each simulation path is given by:

$$
\left.\boldsymbol{P C}(K)_{t=23, T}^{i, j}\right|_{k}=\sum_{m=1}^{M_{\text {time }}} \mathrm{e}^{-\mathrm{r}\left(t_{m}-t\right)} \max \left(\left.\boldsymbol{N}_{t=23, T}^{i, j}\right|_{k, m}-K, 0\right) \cdot P C_{0} d t
$$

where $\boldsymbol{P C}(K)_{t=23, T}^{i, j}$ is a vector of length $M_{p a t h}$, and the expected penalty cost is given by averaging the vector so that:

$P C(K)_{t=23, T}^{i, j}=\left.\frac{1}{M_{\text {path }}} \sum_{k=1}^{M_{\text {path }}} \boldsymbol{P C}(K)_{t=23, T}^{i, j}\right|_{k}$

For the case of no construction, at $t=23$, we have:

$$
C_{t=23}^{N C ; i, j}=P C\left(K_{1}\right)_{t=23}^{i, j}
$$

Next, for the case of expansion, we simplify our problem slightly, by assuming a discrete number of possible modular expansion sizes are available, i.e., $K_{2} \in\left\{K_{2}^{1} K_{2}^{2} K_{2}^{3} \ldots K_{2}^{n}\right\}$, and calculate the expansion cost strategy as:

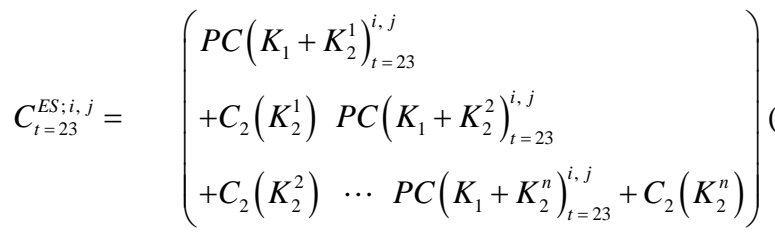

Thus, the value of the cost at each grid point is calculated as:

$v_{t=23}^{i, j}=\min \left(C_{t=23}^{N C ; i, j}, C_{t=23}^{E S ; i, j}\right)$ 

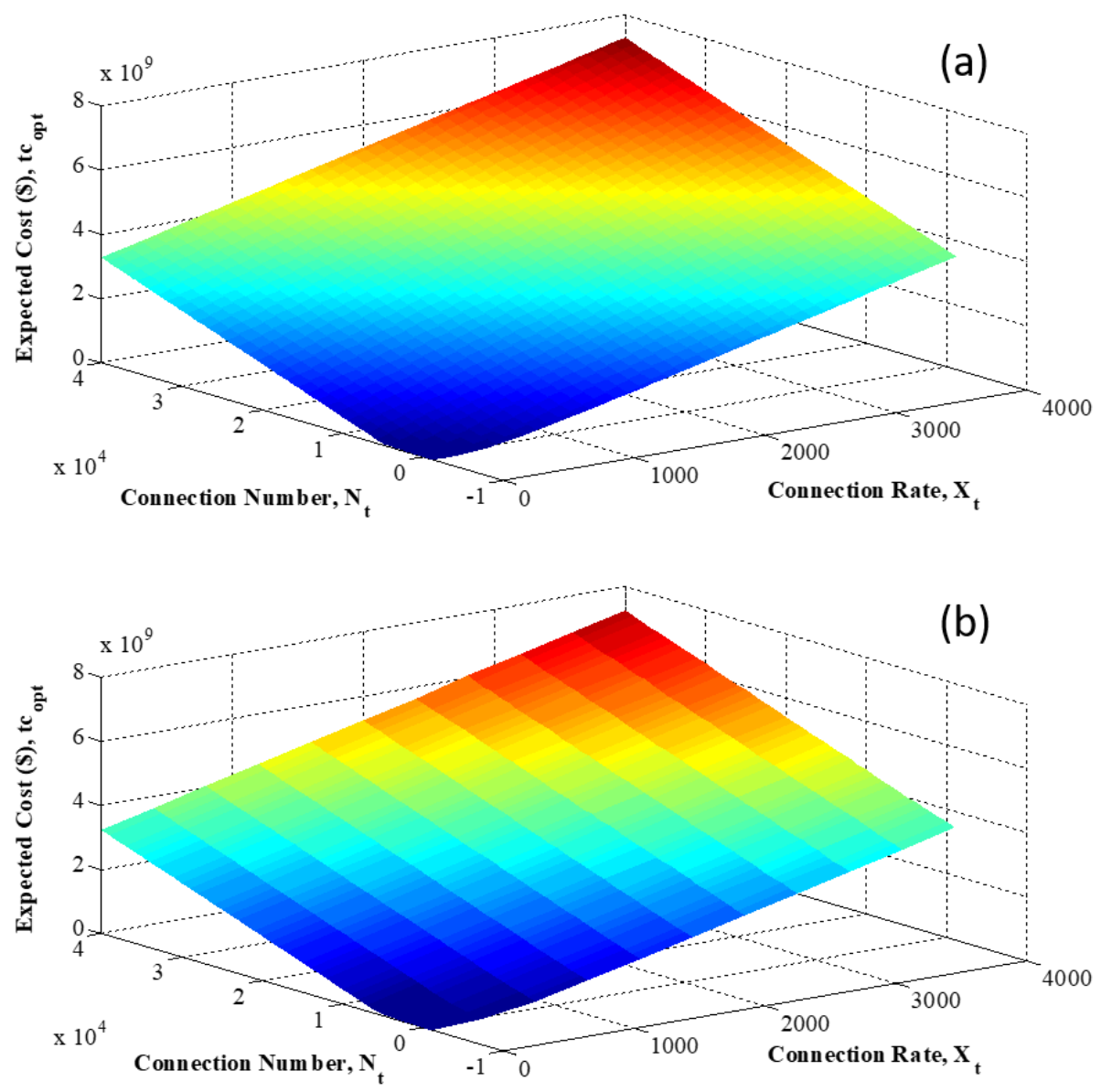

Figure 5. Total cost value plots for the Numerical Model (a) and the Monte-Carlo Simulation (b) at $t=0$.

At the next expansion decision point, $t=22$, we consider two scenarios: the case of no construction and the case of construction. In the case of no construction, we simulate $X_{t}$ from $t=22 y$ to $t=23 y$ to get $\boldsymbol{X}_{t=23, T}^{i, j}$ and $\boldsymbol{N}_{t=23, T}^{i, j}$. We apply Equation (29) to find $\boldsymbol{P C}(K)_{t=23, T}^{i, j}$. Then, for each end point $\left.\boldsymbol{X}_{t=22,23}^{i, j}\right|_{k, M_{\text {time }}}$ and $\left.\boldsymbol{N}_{t=22,23}^{i, j}\right|_{k, M_{\text {ifme }}}$, we use linear interpolation to determine the value of $v_{t=23}^{i, j}\left(\left.\boldsymbol{X}_{t=22,23}^{i, \text { inje }}\right|_{k, M_{\text {time }}},\left.\boldsymbol{N}_{t=22,23}^{i, j}\right|_{k, M_{\text {time }}}\right)$. Thus, the cost for no construction becomes:

$$
\begin{aligned}
C_{t=22}^{N C ; i, j}= & \frac{1}{M_{\text {path }}} \sum_{k=1}^{M_{\text {path }}}\left(\left.P C(K)_{t=23}^{i, j}\right|_{k}\right. \\
& \left.+v_{t=23}^{i, j}\left(\left.\boldsymbol{X}_{t=22,23}^{i, j}\right|_{k, M_{\text {time }}},\left.\boldsymbol{N}_{t=22,23}^{i, j}\right|_{k, M_{\text {time }}}\right) e^{-r(23-22)}\right)
\end{aligned}
$$

For the expansion strategy, we proceed as before, with the key distinction that we simulate $X_{t}$ through the entire remaining time domain, such that we calculate $\boldsymbol{X}_{t=23, T}^{i, j}$ and $\boldsymbol{N}_{t=23, T}^{i, j}$ and utilize Equation (32) to determine $C_{(t=22)}^{(E S ; i, j)}$. Then, Equation (33) is used to determine $v_{t=22}^{i, j}$. The methodology continues in a recursive fashion to $t=2 y$. For $t=0$ to $2 y$ we simply simulate $X_{t}$, determine $N_{t}$ for each path, calculate the interpolated value of $v_{t=2}$ for each case, discount to $t=0$ and calculate the average to find the final value.

\section{Results}

In this section we provide some of the key results of applying the two methods. In particular, we provide a comparison between the Numerical Model (NM) and the Monte Carlo Simulation (MCS). Figures 3 and 4 show the construction boundaries for both the NM and MCS at years 22 and 2, respectively. As can be seen, the two methodologies provide similar results. For the case where $t=2 y$ (Figure 4), the MCS boundary looks slightly different because of the coarser discretization. In Figure 5 we plot the total cost value function as of $t=0$. Again, we see that the results are very similar and an error analysis showed a difference of less than $5 \%$ for all calculated values between the NM and MCS. Finally, in Figure 6 we provide a plot of the total cost as a function of $K_{l}$ and $K_{2 \max }$. This plot can be used by the decision makers to optimize their plant expansion strategy.

\section{Conclusion}

Municipalities that face uncertain growth can benefit by adopting decision making strategies that consider modular expandable design for certain capital investment projects. As we discussed above, the environmental water and wastewater sec- 


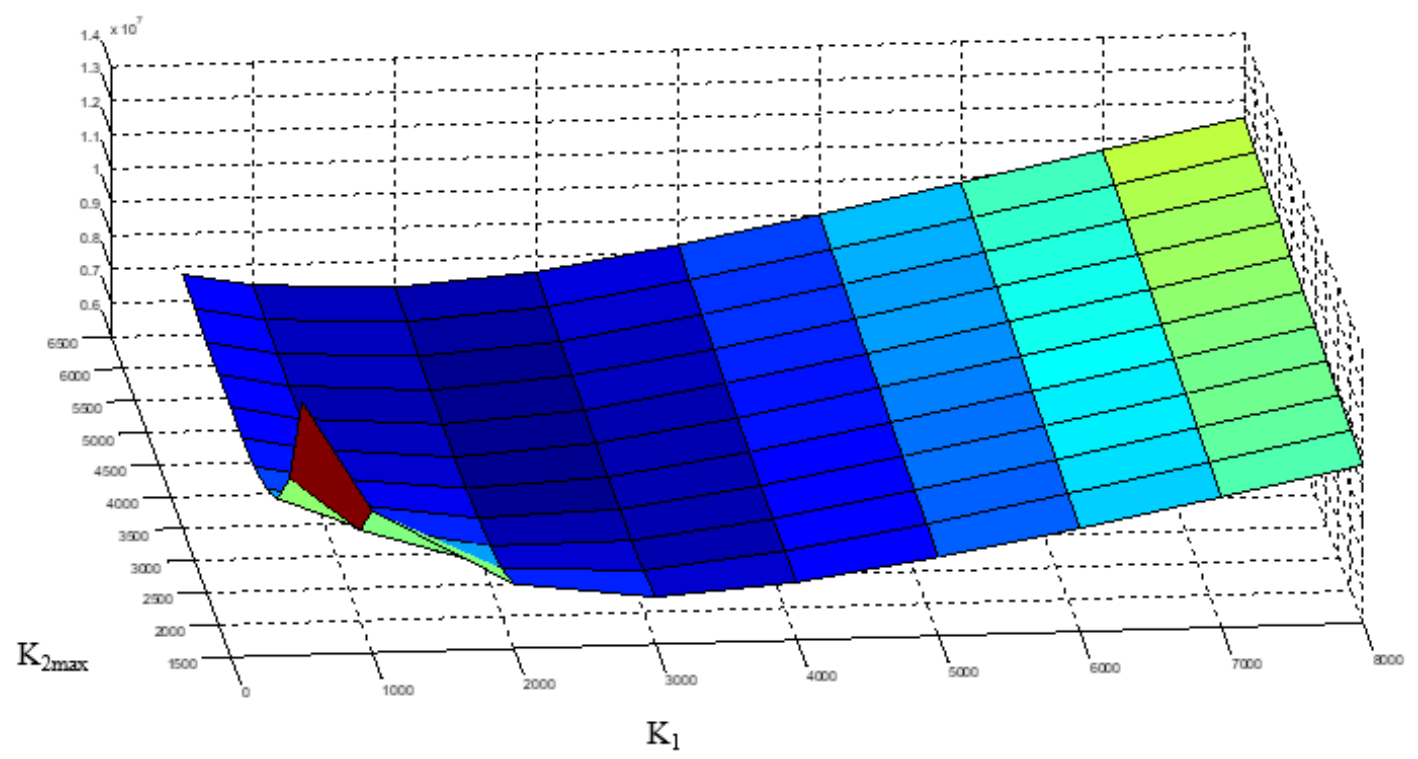

Figure 6. Total cost as a function of $K_{l}$ and $K_{2 \max }$.

tor faces a significant need for near future capital investment. Utilizing size modularity in a wastewater treatment plant development or expansion includes both operational and financial benefits. While financial models that use ROA are known to provide better decision making results than standard models based on DCF analysis, the mathematical complexities associated with even simple scenarios sometimes render ROA modeling an academic exercise with little real world applicability. In this work, we developed a framework to determine the optimal timing and sizing of a two-stage wastewater treatment plant expansion project using two ROA methods, namely 1) a numerical model that requires the solution of a PDE and 2) a model based on Monte-Carlo simulation. The results of the two models showed very good agreement. The numerical model has significant constraints in applicability for real world decision making as it will likely not be easily understood by decision makers and it lacks the flexibility required to model practical scenarios. However, it is a model that is considered to be technically correct and we showed that our more practical simulation based model provides similar results to the numerical model. We thus, have in essence validated our simulation modelling approach. Thus, the simulation methodology presented here can easily be extrapolated to model more realistic scenarios. Furthermore, the model is intoitive, making it significantly more tractable with decision makers.

While the application we presented here was in the context of a wastewater treatment plant capital investment, the methodology can easily be expanded to other capital investment decision making scenarios related to environmental technologies, especially where future demand may be growing but uncertain. For example, consider the case of utilizing wind or solar energy within a growing but uncertain demand context. The option to secure appropriate land today and install limited capacity with the potential for future expansion can be modeled using the approach presented here. Furthermore, the extension of the approach to multiple staged expansions is relatively straightfor- ward. There is significant literature that presents the merits of utilizing ROA for decision making (Trigeorgis 1996, Auger and Guzman, 2010) and optimizing modular design within the environmental framework could produce significant benefits. The methodology presented here strives to fill the gap for assisting with environmental decision-making applications.

Acknowledgments. We would like to thank the NSERC Discovery Grant program for funding.

\section{References}

Auger, F., and Guzmán, J.I. (2010). How rational are investment decisions in the copper industry? Resources Policy, 35(4), 292-300. https://doi.org/10.1016/j.resourpol.2010.07.002

Benedetti, L., Bixio, D., and Vanrolleghem, P.A. (2006). Assessment of WWTP design and upgrade options: balancing costs and risks of standards' exceedance. Water Science and Technology, 54(6-7), 37 1-378. https: //doi.org/10.2166/wst.2006.571

Black, F., and Scholes, M. (1973). The pricing of options and corporate liabilities. Journal of political economy, 81(3), 637-654.

Brennan, M. J., and Schwartz, E.S. (1985). Evaluating natural resource investments. Journal of business, 135-157.

CIRC. (2019). Canadian Infrastructure Report Card: Monitoring the State of Canada's Core Public Infrastructure. http://canadianinfrastructure.ca/downloads/canadian-infrastructure-report-card-2019.pdf.

Antikarov, V., and Copeland, T. (2001). Real options: A practitioner's guide. New York.

Ferrer, J., Seco, A., Serralta, J., Ribes, J., Manga, J., Asensi, E., Morenilla, J.J., and Llavador, F. (2008). DESASS: a software tool for designing, simulating and optimising WWTPs. Environmental Modelling \& Software, 23(1), 19-26. https://doi.org/10.1016/j.envs oft.2007.04.005

Global Cleantech Center. (2013). The US Water Sector On the Verge of Transformation. EY: Building a better working world. Accessed 01 02, 2014. http://www.ey.com/Publication/vwLUAssets/Cleantech _Water_Whitepaper/\$FILE/Cleantech-Water-Whitepaper.pdf.

Lawryshyn, Y., and Jaimungal, S. (2010). Optimization of a municipal 
wastewater plant expansion: A real options approach. Journal of Applied Operations Research, 2(1), 33-42.

Lawryshyn, Y., and Jaimungal. S. (2014). Optimizing a Modular Expansion of a Wastewater Treatment Plant. Civil Engineering and Environmental Systems., 31(3): 243-259. https://doi.org/10.1080/102 86608.2013.853748

Martins, J., Marques, R.C., and Cruz, C.O. (2015). Real options in infrastructure: Revisiting the literature. Journal of infrastructure systems, 21(1), 04014026. https://doi.org/10.1061/(ASCE)IS.1943-

\section{$555 X .0000188$}

Myers, S.C. (1977). Determinants of corporate borrowing. J. Financ. Econ., 5(2), 147-175. https://doi.org/10.1016/0304-405X(77)90015-0

Public-private partnerships in Canada. (2013). Water, Wastewater Sector Study. Public-Private Partnerships Canada. http://www.p3 canada.ca/_files/file/Water\%20Wastewater\%20Study/Water\%20W astewater\%20Sector\%20Study.pdf.

Trigeorgis, L. (1996). Real options: Managerial flexibility and strategy in resource allocation. MIT press. 